\title{
Variations in apparent spatial frequency with stimulus orientation: II. Matching data collected under normal and interferometric viewing conditions
}

\author{
D. O. BOWKER \\ Center for Visual Science, University of Rochester, Rochester, New York 14627
}

\begin{abstract}
Using an adjustment procedure, human otservers matched the apparent spatial frequencies of sinusoidal gratings presented in different orientations $(0,45,90$, and $135 \mathrm{deg})$. Matches were made between all possible pairwise orientation combinations. Significant match deviations indicated that the apparent frequency of a grating depends on its orientation. The most consistent deviations were found between horizontal and vertical gratings, with horizontal gratings appearing to be of a lower spatial frequency than vertical gratings. These effects were relatively independent of stimulus contrast and persisted when the optics of the eye were bypassed with laser interferometry. A neurophysiological explanation of these effects is proposed.
\end{abstract}

There is much evidence, both neurophysiological and psychophysical, that the vertebrate visual system contains mechanisms selective for stimulus orientation. Single units maximally responsive to stimuli in particular orientations have been identified in the cat (Henry, Dreher, \& Bishop, 1974; Hubel \& Wiesel, 1959, 1962) and monkey (Hubel \& Wiesel, 1968; Schiller, Finlay, \& Volman, 1976) cortex, as well as the cortices of other infrahuman species. Psychophysical studies with human observers have shown spatial adaptation (Sekuler, Rubin, \& Cushman, 1968; Sharpe \& Tolhurst, 1973) and spatial masking (Campbell \& Kulikowski, 1966) to be orientation specific. Although one cannot conclude from the psychophysical data that the human visual system contains orientation-selective neurons, it does appear that some prefiltering of spatial stimuli occurs within information channels selective for stimulus orientation.

Further studies have demonstrated the human visual system, and perhaps the visual systems of other species, to respond differentially according to the orientation of a stimulus. Human observers are better able to detect stimuli oriented vertically and horizontally than stimuli oriented obliquely (Appelle,

Thanks are extended to T. Pasternak, for the use of her laboratory facilities and for her helpful suggestions during the course of the research project, and to R. Haber, R. Chapman, W. Makous, R. Springer, and W. Merigan, for their comments and criticisms of the experiments. M. Greenfield was instrumental in the construction of the laser apparatus. This research was funded in part by National Eye Institute Grants EY-01319 (awarded to the Center for Visual Science) and EY-01875 (awarded to T. Pasternak). The author's present address is: Department of Ophthalmology, University of Wisconsin, Room 177, Medical Sciences, Madison, Wisconsin 53706.
1972; Campbell, Kulikowski, \& Levinson, 1966). Stimulus discrimination is likewise superior in the horizontal and vertical meridians (Bowker, 1980b; Matin \& Drivas, 1979; Taylor, 1963). In addition, the apparent contrast (Bowker \& Mandler, 1981) and length (Ritter, 1917; Thompson \& Schiffman, 1974) of a stimulus depends on its orientation.

In a preceding paper (Bowker, 1981), human observers were shown to consistently select horizontal gratings as coarser than vertical gratings in a forcedchoice situation. These variations in apparent spatial frequency with orientation might be due to spatial adaptation effects, small-scale optical aberrations in the cornea or lens, or neuronal anisotropies. The experiments reported in this paper were an attempt to delineate between these alternative explanations. Experiment 1 provides baseline frequency matching data between gratings in different orientations and examines how these data vary with stimulus spatial frequency. Experiment 2 examines the effects of stimulus contrast on the orientation-contingent spatial frequency match deviations. The third experiment is a replication of the first using laser interferometry to produce the stimulus patterns.

\section{EXPERIMENT 1}

\section{Method}

Sinusoidal grating patterns were displayed on two Tektronix 606 oscilloscopes using conventional techniques (for a complete description of stimulus generation procedures, see Bowker and Mandler, 1981). Grating contrast was calibrated ${ }^{1}$ on each oscilloscope to $55 \%$ and the space-averaged luminance of the fields was set to $17.1 \mathrm{~cd} / \mathrm{m}^{2}$. Display orientation was adjusted manually by rotating the oscilloscopes in a pair of specially constructed mounts which guaranteed reliable setting between experimental sessions. The front of the apparatus was masked off so that only 


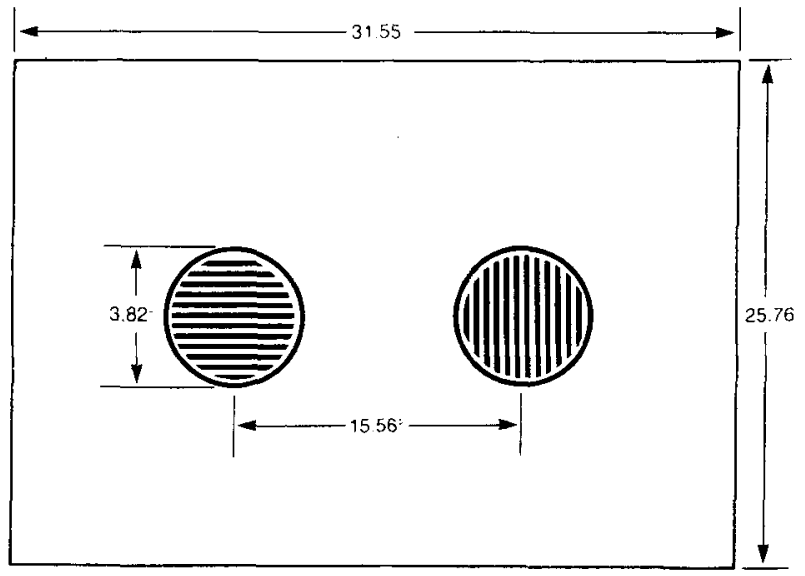

Figure 1. Front-view schematic of display with dimensions of mask given in degrees of visual angle subtended at an observation distance of $114 \mathrm{~cm}$. Actual gratings were sinusoidal, not squarewave as shown.

the central portion of each scope face was visible (Figure 1). Observers viewed the oscilloscopes binocularly at a distance of $114 \mathrm{~cm}$. This distance was fixed by means of a chinrest.

To examine the effect of stimulus orientation on the perceived spatial frequency of sinusoidal gratings, two such gratings were displayed simultaneously on the oscilloscopes and the observer adjusted the frequency of one to match that of the other. These gratings could be presented to the observer at any one of four orientations by correctly positioning the oscilloscope in the rotating jigs. The orientations used were vertical, horizontal, right oblique (45 deg), and left oblique (135 deg). Matches were made between all pairwise orientation combinations, and, from this, a scale of apparent spatial frequency was derived across the various orientations.

In any given data-collection session, only one pairwise comparison of the oriented patterns was tested. Each session consisted of $\mathbf{3 0}$ adjustment trials, half that required the observer to match the frequency on the right oscilloscope to that on the left, and half that required matching of the left frequency to that on the right.

At the beginning of each match trial, the observer heard one of two tones indicating which oscilloscope frequency was to be adjusted and which was to serve as the standard. "Test frequency" will hereafter refer to the frequency of the grating that is being adjusted by the observer, and "standard frequency" will refer to the frequency that the observer is required to match. Grating patterns were displayed for $3 \mathrm{sec}$ or until the observer pressed one of three hand-held switches. One button increased the test frequency by $1.0 \%$, one decreased it by $1.0 \%$, and the third signaled that the subject was satisfied with the match. To remove some of the inherent predictability in the adjustment procedure, the buttons that changed the frequency did so only $70 \%$ of the time, having no effect the other $30 \%$ of the time. This was randomly determined by the computer program on a trial-by-trial basis. In addition, the initial test frequency was chosen from one of 19 values about the standard frequency. Nine of these values were above the standard, nine were below, and one was identical. The frequency increment between adjacent values was $1.0 \%$ spatial frequency of the standard. After each button press, the screen reverted to the space-averaged luminance homogeneous field for s sec; then the next stimulus was presented. This procedure was repeated several times until the subject pressed the third button indicating a match. The final value was recorded by the computer, and the next trial was presented after a 7-sec interval. A session consisting of $30 \mathrm{such}$ trials typically lasted $40-50 \mathrm{~min}$.
To reduce any side biases on the part of the observer, the side of stimulus orientation was counterbalanced between sessions. Therefore, 60 adjustments were made for each orientation combination at each spatial frequency of the standard (600 adjustments per frequency). Average match deviations were computed for each combination. A scale of apparent spatial frequency was obtained for each orientation of the standard by examining match deviations of test gratings in other orientations when compared with that standard. Since stimuli could appear in any one of four orientations (vertical, horizontal, right oblique, left oblique), four scales were obtained for each frequency of the standard.

\section{Results}

Data obtained in this fashion are shown in Figure 2 for Observer D.O.B. ${ }^{2}$ The standard frequency in this case was 8.0 cycles $/ \mathrm{deg}$. The four plots in this figure represent direct comparisons made with each orientation of the standard. In each case, the horizontal axis represents the expected values if there

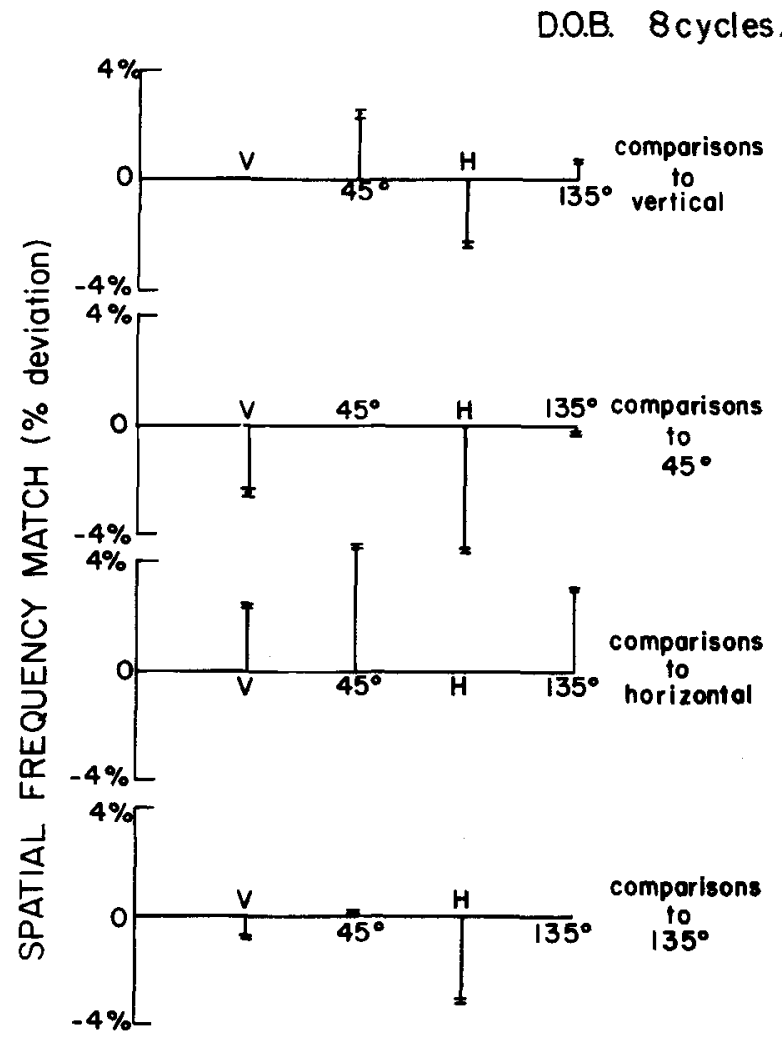

\section{ORIENTATION}

Figure 2. Spatial frequency match deviations given in percent error from the $8.0 \mathrm{cycles} / \mathrm{deg}$ standard. Deviations are represented as a function of test orientation for four standard orientations. The horizontal axis represents the expected value if no match deviations occurred. Points above this axis represent matches lower in frequency than the standard (indicating a higher apparent frequency than the standard), and points below the axis represent matches higher than the standard frequency. Error bars are the $99 \%$ confidence intervals for the population means. Observer D.0.B. 
were no deviations in the spatial-frequency matches. Deviations above the line indicate that the gratings in this particular orientation looked higher in spatial frequency ("finer") than the standard spatial frequency, while deviations below the line indicate that gratings in these orientations looked lower in spatial frequency ("coarser") than the standard. In the uppermost graph, for example, the horizontal grating looked lower in frequency than the standard vertical grating. That is, the horizontal grating had to be adjusted to a higher spatial frequency to match the standard.

It is evident from these graphs that the oblique gratings tend to look higher in spatial frequency than the others and that horizontal gratings look coarser than the others. Not only is the variability in these data low (error bars represent the $99 \%$ confidence intervals for the population mean), but a similar picture emerges from each set of comparisons.

These four scales were collapsed by normalizing all match deviations to the vertical. Additional data obtained at other frequencies of the standard $(.95$, $4.0,8.0$, and 12.0 cycles $/ \mathrm{deg}$ ) were also normalized to the vertical orientation. These data are summarized in Figure 3. Normalized deviations in the various orientations are plotted as a function of the spatial frequency of the standard. All deviations shown in Figure 3 are statistically reliable at the $p<.01$ level of significance. Details of the analysis are provided in the Appendix.

As can be seen in Figure 3, horizontal gratings appear coarser than vertical gratings at all frequencies of the standard. The magnitude of this effect is between $2.0 \%$ and $3.5 \%$. At the two lower frequencies of the standard, the obliques look coarser than the vertical gratings, but at the two higher frequencies, they look finer.

\section{D.O.B.}

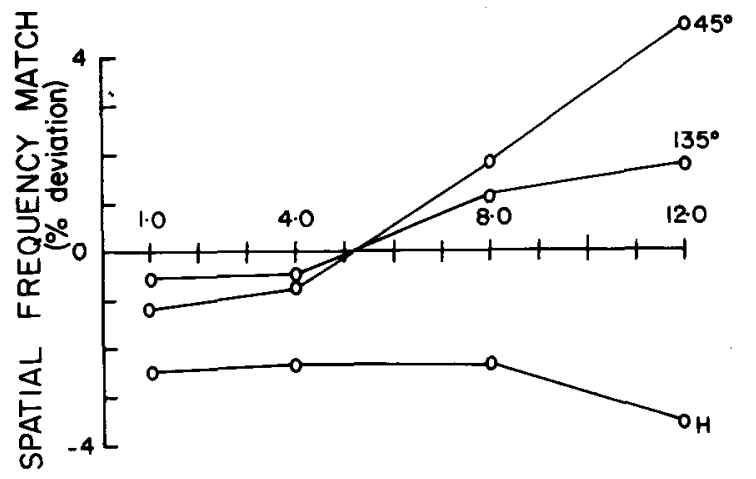

$$
\begin{gathered}
\text { SPATIAL FREQUENCY } \\
\text { OF STANDARD } \\
\text { (cycles per degree) }
\end{gathered}
$$

Figure 3. Scaled match deviations given as a function of standard spatial frequency for 45,90 (horizontal), and 135 deg. $\mathrm{Ob}$ server D.0.B.
Average match deviations for those conditions in which test and standard gratings were presented in the same orientation were consistently less than $.3 \%$ for this observer.

\section{Discussion}

The data of Experiment 1 are consistent with those reported for a large number of observers in a preceding paper (Bowker, 1981). In that paper, 53 observers were shown a series of grating pairs, one grating of which was horizontal and the other vertical. The observers had to choose which of the two gratings was coarser (i.e., had a lower spatial frequency). Most subjects tested selected the horizontal gratings as being coarser on the majority of presentations in which the two were actually identical in spatial frequency.

These results are certainly not the first reported instances of variations in the appearance of grating spatial frequency. Virsu (1974), using a matching technique, found that dark adaptation had an influence on apparent spatial frequency. Gratings that fell on a light-adapted region of the retina appeared to be coarser than gratings of the same spatial frequency that fell on a dark-adapted region. Virsu (1974) postulated that in the dark-adapted region, retinal neurons with concentric receptive field organizations were receiving a diminished input from their inhibitory surrounds (Barlow, Fitzhugh, \& Kuffler, 1957) and therefore had a broader spatial profile than similar neurons located in the light-adapted region. Cells that responded to high spatial frequencies in the light-adapted state became maximally sensitive to lower frequencies in the dark-adapted state. When a grating of a given spatial frequency was projected to light- and dark-adapted regions of the retina, it was received by two distinctly different populations of neurons. The population in the dark-adapted region normally responded to higher spatial frequencies, while the population in the light-adapted region typically responded to lower spatial frequencies. Virsu (1974) assumed that spatial frequency perception arose from the activation of specific channels in the visual system. The fact that the same spatial frequency stimulated different neural populations in the two regions of the retina meant that the two gratings would appear to have different spatial frequencies.

In a subsequent paper, Virsu and Nyman (1974) demonstrated that the temporal modulation of a grating stimulus could affect its apparent spatial frequency. At high rates of temporal modulation, the apparent spatial frequency of gratings increased. Virsu and Nyman (1974) explained the frequency shifts as resulting from a reduction in the effect of surround inhibition brought about by the temporal modulation of the stimulus.

Another factor known to affect the perceived spatial frequency of sinusoidal gratings is the observ- 
er's state of spatial adaptation. Several studies (Blakemore \& Nachmias, 1971; Blakemore, Nachmias, \& Sutton, 1970; Blakemore \& Sutton, 1969) have demonstrated that prior adaptation to a high-contrast grating pattern will cause subsequently viewed gratings to appear shifted in frequency. Gratings finer than the adaptation grating will appear finer still, and gratings coarser than the adaptation grating will appear coarser than they actually are. Gratings of the same frequency as the adapting stimulus will not shift to either higher or lower apparent frequencies.

Further examinations of these frequency shifts following spatial adaptation have shown them to be orientation selective. As the orientation disparity between the test and adaptation was increased, the magnitude of the spatial frequency shift decreased (Blakemore \& Nachmias, 1971; Blakemore, Nachmias, \& Sutton, 1970). Initial reports went so far as to claim "a horizontal adapting grating has no effect on vertical test gratings" (Blakemore, Nachmias, \& Sutton, 1970) when, in fact, no such tests were reported. Data shown in these initial papers did not actually include orientation disparities as great as $90 \mathrm{deg}$.

In a recent paper, Heeley (1979) claims to have found spatial frequency shifts when test and adaptation gratings differed by as much as $90 \mathrm{deg}$. However, Heeley's (1979) methods are questionable, particularly in light of the data presented in Experiment 1 and in the previous paper (Bowker, 1981). These difficulties are discussed in greater detail in the preceding paper and in Wenderoth (1980). Despite the problems in the Heeley (1979) study, the results raise the possibility that the orientationcontingent distortions in perceived spatial frequency described in Experiment 1 derive from adaptation effects. Stimuli used in the experiments discussed above were presented at very high contrast levels $(55 \%)$. Previous studies have shown that frequency shifts following adaptation are large at high contrast levels and diminish at lower contrasts (Blakemore, Nachmias, \& Sutton, 1970; Heeley, 1979). If the spatial frequency distortions described in Experiment 1 arise from spatial frequency adaptation, they should decrease as the contrast of the stimulus is decreased.

\section{EXPERIMENT 2}

\section{Method}

Stimulus generation and calibration procedures were identical to those used in Experiment 1. The one exception to this was that stimulus contrast was now calibrated to one of three values: $55.0 \%$, $20.0 \%$, and $5.5 \%$. Again, the contrast was identical on the two oscilloscopes and space-averaged luminance was calibrated to $17.1 \mathrm{~cd} / \mathrm{m}^{2}$.

To examine the effect of stimulus contrast on the spatial frequency match deviations, Observer D.O.B. made a number of adjustments between horizontal and vertical gratings presented at one of three contrast levels. The matching procedure used was identical to that described in Experiment 1 . Thirty matches were made in each session, and side of stimulus orientation was counter-

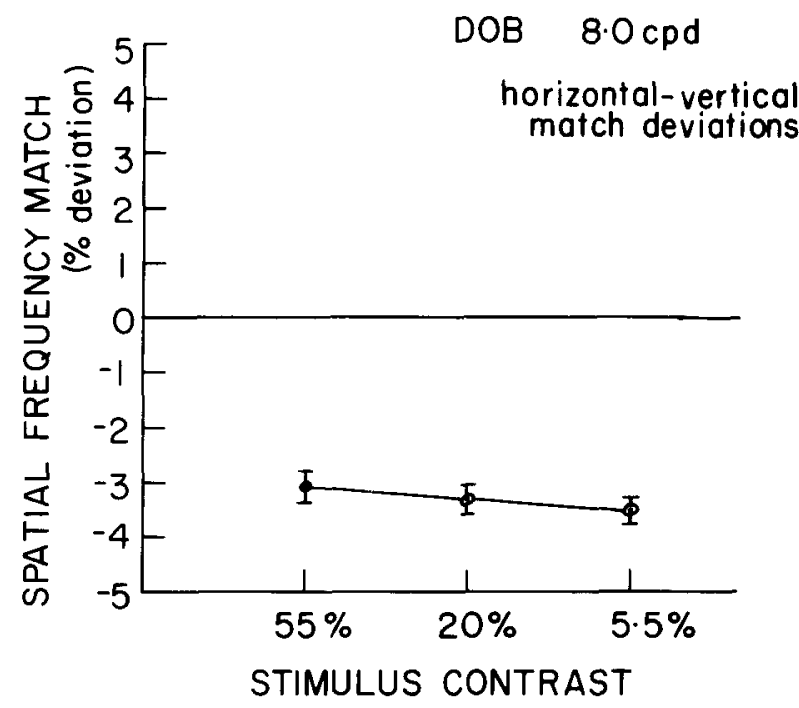

Figure 4. Horizontal-vertical frequency match deviations at three contrast levels. Points below the horizontal axis indicate that horizontal gratings looked coarser than vertical gratings. Error bars are standard errors. Observer D.0.B.

balanced between sessions to yield 60 adjustments for each contrast level. Stimulus spatial frequency was, in all cases, 8.0 cycles/ deg.

\section{Results}

Results of this experiment are shown in Figure 4. As can be seen, contrast had very little effect on the frequency match deviations between horizontal and vertical gratings. An analysis of variance was performed on this data to determine whether or not horizontal-vertical frequency match deviations varied with stimulus contrast. The main effect of contrast was found to be statistically insignificant $[F(2)=.93]$.

\section{Discussion}

The results of the first and second experiments cannot easily be explained by the spatial adaptation hypothesis. If the orientation-contingent distortions of apparent spatial frequency were due solely to spatial adaptation, they should have diminished as stimulus contrast was decreased. This was not found to be the case. Furthermore, the fact that spatial adaptation effects are orientation contingent predicts that larger match deviations will occur between oblique and vertical gratings than between horizontal and vertical gratings. Results of the first experiment indicate that horizontal-vertical match deviations are larger than oblique-vertical matches, a result that is hard to reconcile with the adaptation hypothesis.

The lack of a contrast effect in Experiment 2 also suggests that afterimages were not responsible for the spatial frequency match deviations. Afterimages are known to increase with stimulus contrast (Brown, 1965). The subject did not observe afterimages in any of the experimental conditions. 
An alternative explanation of the results involves small-scale optical aberrations in the cornea or lens. If either of these structures were astigmatic, it could explain the anisotropies in apparent spatial frequency. This explanation can be directly tested with laser interferometry. Such a test was performed in Experiment 3.

\section{EXPERIMENT 3}

\section{Method}

Sinusoidal gratings were imaged directly on the retina of the observer's right eye with laser interference techniques. The laser interferometer, ${ }^{3}$ shown in Figure 5, consists of a $500-\mathrm{mW}$ heliumneon laser (Spectra Physics Model 155) projecting through expander lens $L_{1}$ to beamsplitter $B_{1}$. This beamsplitter divides the incoming signal into two components which are reimaged by mirrors $M_{1}$ and $M_{2}$ and beamsplitter $B S_{2}$ to pass through the Maxwellian lens, $L_{2}$, into the observer's pupil. If the beamsplitters and mirrors are properly aligned, such that the two beams pass through slightly disparate locations in the pupil, a sinusoidally modulated grating is produced on the observer's retina. The intensity of the stimulus may be reduced by placing neutral density filters in filter box ND; the orientation of the test stimulus is varied by rotating dove prism DP. Stimulus spatial frequency is controlled by changing the position of mirror $\mathbf{M}_{\mathbf{3}}$. As the beams are made to diverge, spatial frequency increases. As they converge, frequency de-

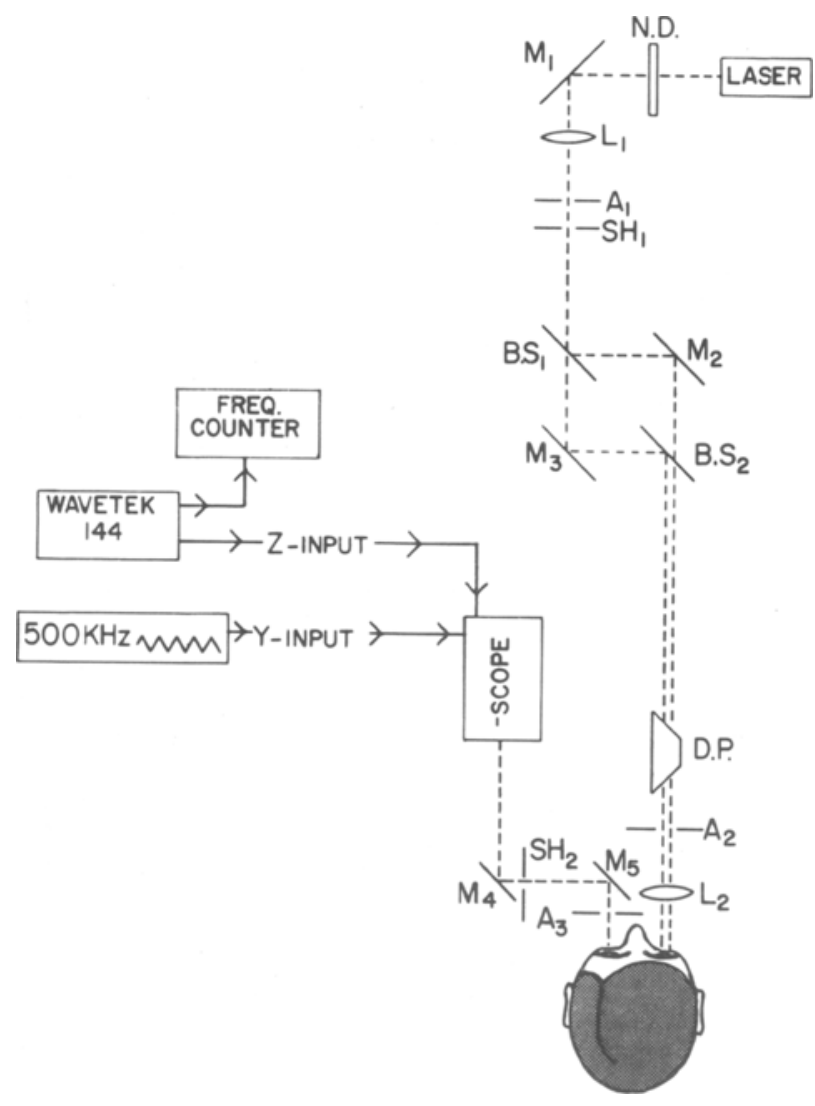

Figure 5. Dichoptic laser interferometer. Subject views interferometric grating through right eye and oscilloscope grating through left eye. creases. Circular aperture $A_{2}$ is positioned to remove any edge artifacts present in the stimulus. In all experiments, stimulus luminance was $8.6 \mathrm{~cd} / \mathrm{m}^{2}$. This was measured at the exit pupil of the Maxwellian lens with a Spectra spotmeter. The contrast of interferometrically produced gratings is theoretically $100 \%$.

Through the second channel of the optical system, which essentially consists of two mirrors $\left(\mathbf{M}_{4}\right.$ and $\left.\mathbf{M}_{5}\right)$ and a circular aperture (both apertures were $2 \mathrm{deg}$ in diameter), the observer's left eye was able to view a sinusoidal grating pattern displayed on an oscilloscope face (Tektronix 604). This grating pattern was produced by sending a phase-locked $500-\mathrm{kHz}$ triangular waveform into the scope's $\mathrm{Y}$ amplifier and a sinusoidally modulated waveform into the $\mathrm{Z}$ amplifier. The sinusoidal waveform was produced by a Wavetek 144 oscillator, the output of which was also fed into a digital frequency counter (Heath Schlumberger Model SM-118A). The sinusoidal signal from the Wavetek oscillator was synchronized to the oscilloscope's internal time base. Mean luminance of the grating pattern was $8.6 \mathrm{~cd} / \mathrm{m}^{2}$, and its contrast was calibrated to $55.0 \%$. The oscilloscope pattern was always vertically oriented and was observed at a distance of $73.7 \mathrm{~cm}$. The observer's head position was held constant by means of a fitted bite-bar constructed of dental impression wax.

Shutters $\mathrm{Sh}_{1}$ and $\mathrm{Sh}_{2}$ (Uniblitz Corporation), placed in the interferometer and oscilloscope light paths, were under the control of the observer. The observer also controlled the frequency of the waveform produced on the oscilloscope screen.

The subject would briefly (approximately $1 \mathrm{sec}$ ) view the interferometrically produced grating and then switch his gaze to the oscilloscope grating. This was accomplished by opening and closing shutters $\mathrm{Sh}_{1}$ and $\mathrm{Sh}_{2}$. The subject would then adjust the frequency of this grating to match that of the other. The subject repeated these observations until he was satisfied that the two gratings matched in apparent spatial frequency. He then verbally signaled his satisfaction to the experimenter, who, in turn, recorded the oscillator output frequency. The experimenter then reset the dial of the oscillator to produce a grating well above or below (on alternate trials) the interferometric frequency and signaled the subject to proceed with the next match. Such adjustments were made to interferometric gratings imaged vertically, horizontally, and at the two obliques ( 45 and $135 \mathrm{deg}$ ).

\section{Results}

Average match deviations are shown at a number of standard frequencies in Figures 6 (Observer D.O.B.) and 7 (Observer A.N.P. ${ }^{4}$ ). Each point in Figure 6 is based on the mean of 16 adjustments. Between 4 and 8 adjustments were taken for each point in Figure 7. Results for D.O.B. are similar to those reported in Experiment 1 (Figure 3). At all standard frequencies, horizontally oriented gratings appeared coarser than vertically oriented gratings. Oblique gratings appeared coarser than vertical gratings at the lowest frequency of the standard ( $2.75 \mathrm{cycles} / \mathrm{deg})$ and finer at the two higher frequencies (6.69 and 8.06 cycles/deg). Data from A.N.P. replicated the essential aspects of these results.

Arguing that these data indicate a postoptical basis for the orientation-contingent deviations in apparent spatial frequency requires that certain assumptions be true. The most important assumption in this interpretation is that the frequency of interferometrically produced gratings is independent of the optics of the cornea and lens. To test this assumption, control observations were made with astigmatic lenses of various powers placed in the interferometric channel. 


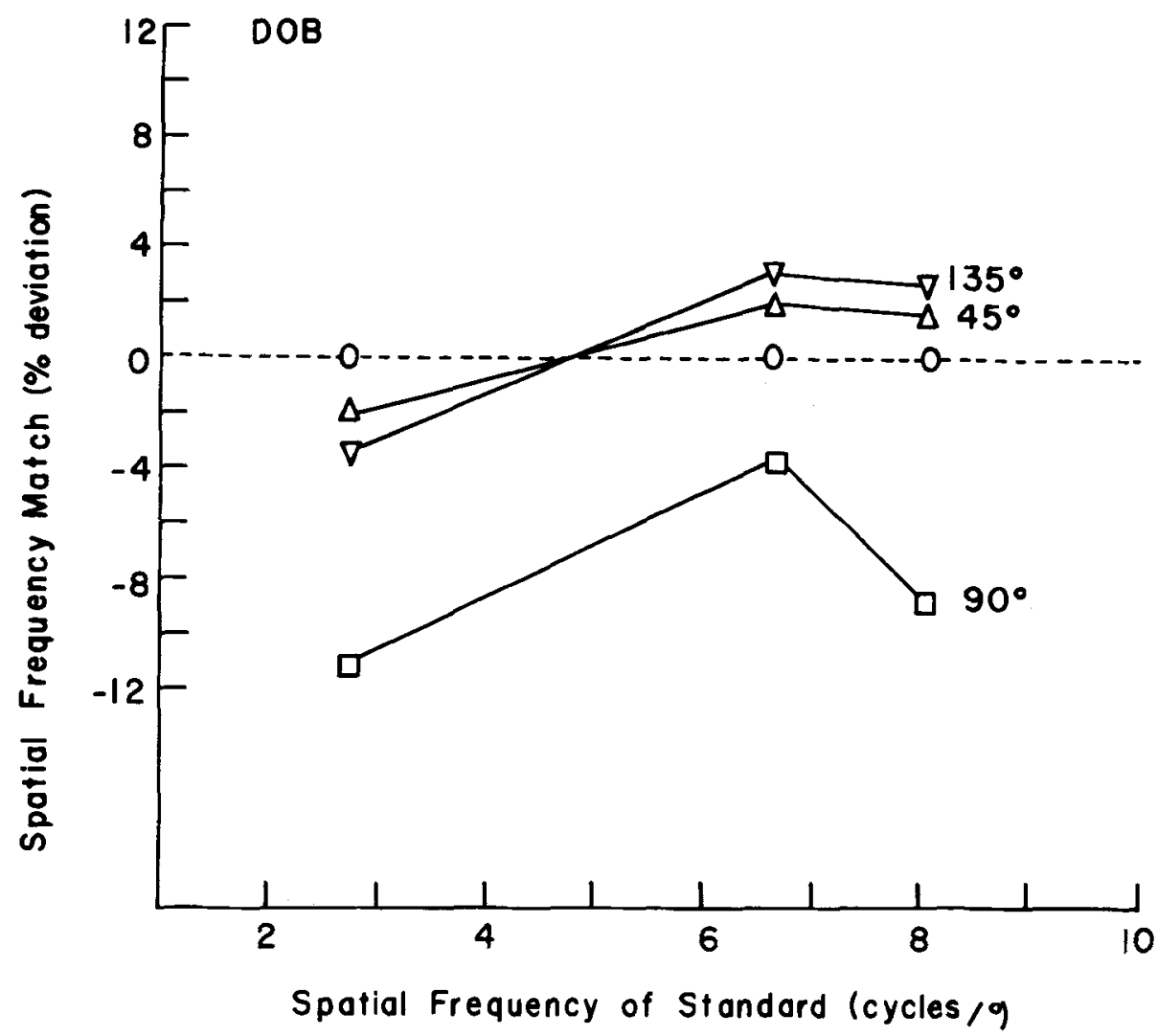

Figure 6. Scaled frequency match deviations as a function of standard spatial frequency for three orientations. Matches obtained at test orientations of 45 deg (upward triangles), 90 deg (squares), and 135 deg (downward triangles) are plotted relative to vertical matches (open circles). Data collected with laser interferometer. Observer D.O.B.

These lenses were placed next to the final aperture on the side opposite to the observer. In this way, the lenses would have no effect on the appearance of the aperture itself.

Spatial frequency adjustments were made to vertical gratings imaged through either no lens or a $+1-$, $+2-$, or +3-diopter astigmatic lens. These lenses were oriented with their plane of maximal magnification either in the horizontal meridian or in the vertical meridian. Eight adjustments were made in each of these conditions. These conditions were randomly intermixed in sets of two trials each.

Results (Figure 8) indicate that the astigmatic lenses do have a profound effect on the perceived spatial frequency of the interferometric gratings. As the power of the lens increases, the effect of its orientation increases. At first glance, these data appear to cloud the issue substantially. If an astigmatic lens can affect the perceived spatial frequency of the interferometric gratings, how can one establish the degree to which optical influences affect spatial frequency judgments in the normal situation?

A more detailed examination of the data, however, points to the following conclusion. If the optics of the cornea or lens had any influence on the earlier matching data of Observer D.O.B. (Figure 3 and 6), they served only to minimize the spatial frequency match deviations and could not be totally responsible for them. In comparing spatial frequency judgments made under natural viewing conditions with those made under conditions of laser interferometry, a perfect test case is provided for examining the hypothesis that any orientation-contingent spatial frequency match deviations are caused by optical aberrations. Due to the nature of laser interference, if the effects in question result from optical factors alone, results collected under natural conditions and interference conditions should run in opposite directions. Why is this so?

An astigmatic lens is, by definition, a lens with a greater magnification in one meridian than in another. If one views a stimulus through such a lens, the appearance of the stimulus will depend on the orientation of the lens. If the lens is oriented with its maximal power in the vertical meridian, objects will appear to stretch vertically. A horizontal grating would look coarser than a vertical grating in this instance. Looking through the same lens with the major power in the horizontal meridian will stretch images horizontally. A vertical grating would now appear 


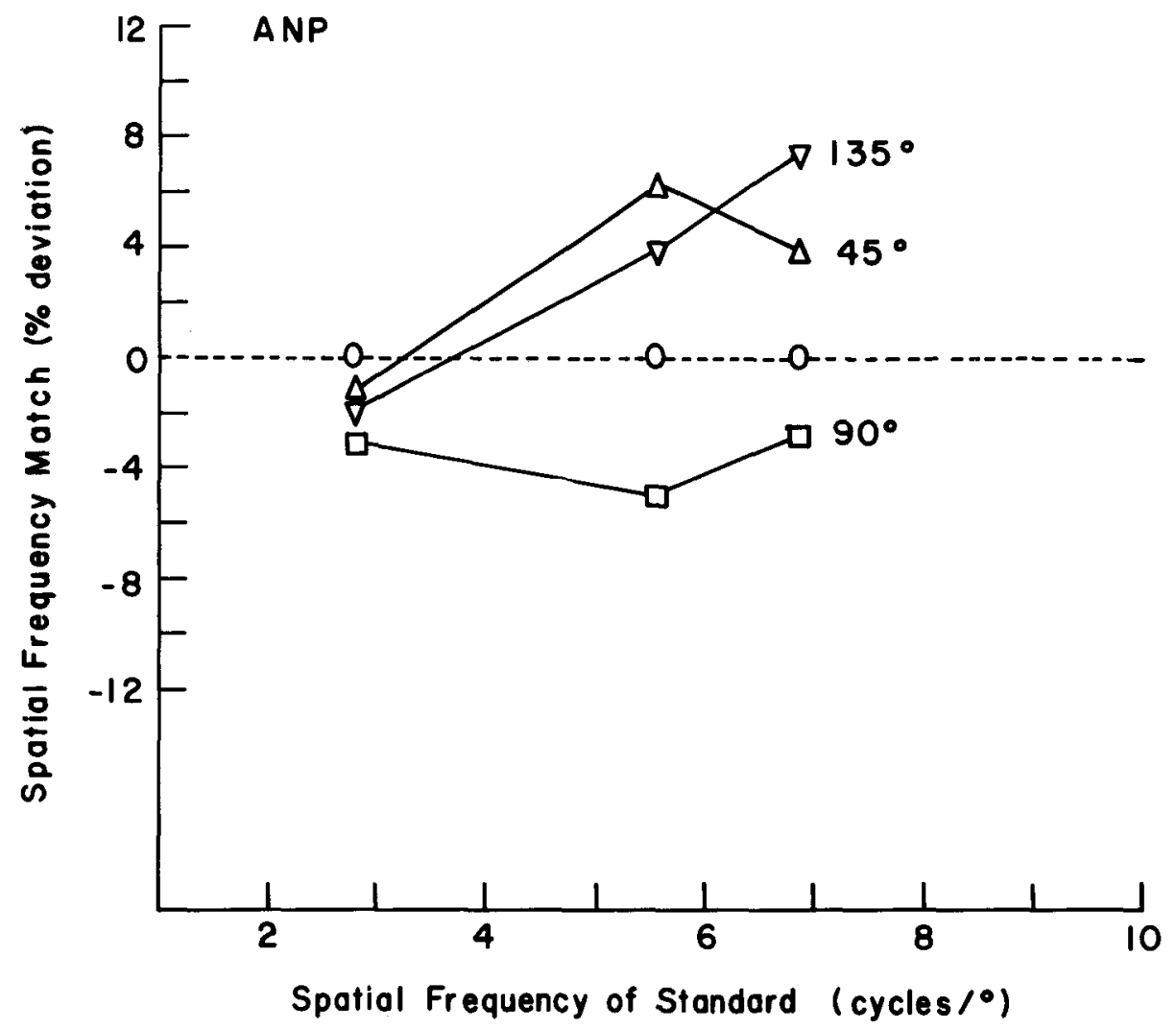

Figure 7. Same as Figure 6. Observer A.N.P.

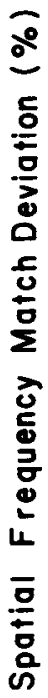

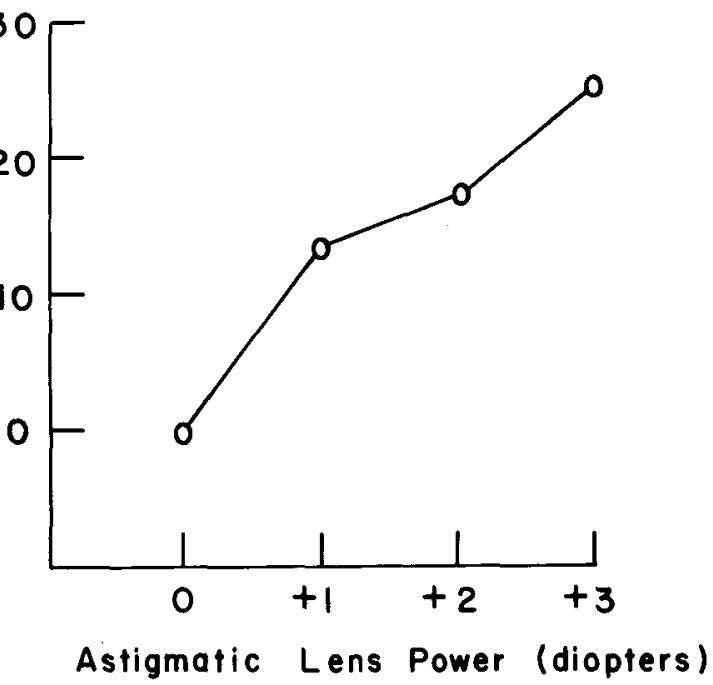

Figure 8. Spatial frequency match deviations for different powers of astigmatic lenses placed in interferometric channel. Deviations calculated by dividing average frequency matches made with major lens power in horizontal meridian by matches made with major power in vertical meridian. All gratings were vertically oriented. Observer D.O.B. coarser than a horizontal grating. However, if one inserts this lens in the laser interference beam, the opposite effects will occur. In the interferometer, the orientation of a grating is determined by the relative locations of the two coherent beams. To produce a vertical grating, the beams must be placed laterally (a horizontal displacement) to one another. Moreover, diverging these beams causes the frequency to increase and converging them causes the frequency to decrease. If a vertical grating is being produced, placing an astigmatic lens in the beam path with the major power oriented horizontally will cause the beams to diverge, resulting in a spatial frequency increase. Note that this effect is opposite to that which one gets while viewing an object naturally through such a lens.

The fact that the results collected under natural viewing conditions are similar to those collected using the laser interferometer strongly indicates that these effects are not caused by optical influences alone. A postoptical anisotropy in the visual system must be responsible for the orientation-contingent deviations in apparent spatial frequency.

\section{DISCUSSION AND CONCLUSIONS}

Data presented in this paper and in the preceding one (Bowker, 1981) show that the apparent spatial 
frequency of a grating is dependent on the orientation of the grating. The most consistent result is that horizontal gratings appear coarser than vertical gratings of the same spatial frequency. This horizontalvertical discrepancy is evident in a majority of observers tested, is essentially independent of stimulus spatial frequency and contrast, and persists when the optics of the eye are bypassed with laser interferometry. Additional experiments (Bowker, 1980a), not presented, show that these effects are not dependent on the relative arrangements of the oscilloscope displays or on the inclination of the observer (that is, the match deviations are relative to the observer's orientation and not necessarily to the gravitational orientation).

Some explanations of the orientation-contingent differences in apparent spatial frequency may be rejected on the basis of the data presented. For instance, these differences cannot be solely dependent on optical aberrations in the cornea or lens. As mentioned above, the spatial frequency match deviations persist as the optics of the eye are bypassed with laser interference techniques. Also, if optical aberrations are responsible, a large percentage of human observers would have to have such aberrations in a particular meridian.

It is also unlikely that these effects derive from spatial adaptation. First, in virtually all of the matching experiments, any adaptation effects would presumably be randomized by the random presentation of test frequencies above and below the standard frequency. Why shifts would consistently occur in one direction and not the other would have to be explained by the adaptation model. Second, spatial frequency shifts following adaptation are contingent upon stimulus orientation. Whether or not such effects occur with orientation disparities greater than $30 \mathrm{deg}$ is open to debate, but, regardless, frequency shifts are certainly of their largest magnitude when the test and adaptation gratings are presented in the same orientation. If the frequency match deviations reported were due only to adaptation effects, the largest match deviations should have occurred when the two gratings were presented in the same orientation and not when they were presented with orientation disparities of 45 or $90 \mathrm{deg}$. This was not the case. Third, the fact that horizontal-vertical match deviations are considerably different from 45-135-deg matches requires additional ad hoc assumptions in an adaptation explanation. Fourth, an adaptation explanation predicts that the frequency match deviations would decrease as the stimulus contrast decreased. Data presented in Experiment 2 show that this is not true for horizontal-vertical matches. Test contrast had no measurable effect on the spatial frequency match deviations. Finally, the results of the preceding paper (Bowker, 1981) showed that most observers tested reported horizontal gratings as coarser than vertical gratings almost immediately following stimulus exposure, presumably before their visual systems adapted to the grating patterns. From these considerations, it seems that spatial adaptation cannot adequately explain all aspects of the data.

If one is willing to make a number of assumptions, it is possible to model horizontal-vertical differences in spatial frequency appearance from anisotropic retinal receptive fields. If receptive fields of cells in the human retina are elongated in the horizontal meridian, as they are in the rabbit (Levick, 1967) and turtle (Normann, Kolb, Hanani, Pasino, \& Holub, 1979) retina, it is probable that a given unit would be most responsive to a large grating when the grating was vertically oriented and a small grating that was oriented horizontally. By the same reasoning, if one were to project two gratings of the same spatial frequency, one being horizontally oriented and the other being vertically oriented, onto such an anisotropic detector, the vertical grating would be maximally received by a neuron with a smaller receptive field than the horizontal grating. Using a "labeledline" approach similar to that forwarded by Virsu (1974), the larger receptive field would be part of a neural channel signaling the presence of a large object to the brain while the smaller receptive field would be part of a neural channel signaling the presence of a smaller object to the brain. Even though the physical sizes of the two gratings were the same, the vertical grating would be interpreted as smaller (of a higher spatial frequency) than the horizontal grating. This model rests on assumptions not currently based on solid empirical grounds. Further assumptions are required to deal with the oblique match deviations and the significant interaction between stimulus orientation and spatial frequency.

Whatever the explanation, the data presented in this and the preceding paper (Bowker, 1981) have definite implications for the interpretation of other experiments. The apparent spatial frequency of a grating is not isotropic, as has been assumed (Heeley, 1979), but varies with stimulus orientation. In addition, data presented in the third experiment suggest that although the contrast of an interferometric grating is independent of optical influences, the frequency of such a grating is not. Since contrast sensitivity is known to vary with spatial frequency, detection thresholds determined with laser interferometry may not be completely independent of optical factors in the observer's cornea and lens.

\section{REFERENCES}

Appelte, S. Perception and discrimination as a function of stimulus orientation: The "oblique effect" in man and animals. Psychological Bulletin, 1972, 78, 266-278.

Barlow, H. B., Fitzhugh, R., \& Kuffler, S. W. Change of organization in the receptive fields of cat's retina during dark adaptation. Journal of Physiology (London), 1957, 137, 338-354. 
Blakemore, C., \& Nachmias, J. The orientation specificity of two visual aftereffects. Journal of Physiology (London), 1971, 213, 157-174.

Blakemore, C., Nachmias, J., \& Sutton, P. The perceived spatial frequency shift: Evidence for frequency-selective neurons in the human brain. Journal of Physiology (London), 1970, 210, 727-750.

Blakemore, C., \& Sutron, P. Size adaptation: A new aftereffect. Science, 1969, 166, 245-247.

BOWKER, D. O. The effect of orientation on the appearance and discriminability of suprathreshold gratings. Unpublished doctoral thesis, University of Rochester, 1980. (a)

Bowker, D. O. Spatial frequency discrimination thresholds in different orientations. Journal of the Optical Society of America, $1980,70,462-463$. (b)

BowkER, D. O. Variations in apparent spatial frequency with stimulus orientation. 1. Incidence of the effect in the general population. Perception \& Psychophysics, 1981, 29, 563-567.

Bowker, D. O., \& MANDler, M. B. Apparent contrast of suprathreshold gratings varies with stimulus orientation. Perception \& Psychophysics, 1981, 29, 585-588.

Brown, J. L. Afterimages. In C. H. Graham (Ed.), Vision and visual perception. New York: Wiley, 1965.

Campbell, F, W., \& Kulikowski, J. J. Orientational specificity of the human visual system. Journal of Physiology (London), $1966,187,437-445$.

Campbell, F. W., Kulikowsi, J. J., \& Levinson, J. The effect of orientation on the visual resolution of gratings. Journal of Physiology (London), 1966, 187, 427-436.

HeEley, D. W. A perceived spatial frequency shift at orientations orthogonal to adapting gratings. Vision Research, 1979, 19. $1229-1236$.

Henky, G. H., Dreher, B., \& Bishop, P. O. Otientation specificity of cells in cat striate cortex. Journal of Neurophysiology, $1974,37,1394-1409$.

HuBEL, D. H., \& Wiesel, T. N. Receptive fields in single neurones in the cat's striate cortex. Journal of Physiology (London), $1959,148,574-591$.

HubeL, D. H., \& Wiesel, T. N. Receptive fields, binocular interaction and functional architecture in the cat's visual cortex. Journal of Physiology (London), 1962, 160, 106-154.

HubeL, D. H., \& Wiesel, T. N. Receptive fields and functional architecture of monkey striate cortex. Journal of Physiology (London), 1968, 198, 215-243.

LEvick, W. R. Receptive fields and trigger features of ganglion celis in the visual streak of the rabbit's retina. Journal of Physiology (London), 1967, 188, 285-307.

Matin, E., \& Drivas, A. Acuity for orientation measured with a sequential recognition task and signal detection methods. Perception \& Psychophysics, 1979, 25, 161-168.

Normann, R. A., Kolb, H., Hanani, M., Pasino, E., \& Holub, R. Orientation of horizontal cell axon terminals in the streak of the turtle retina. Nature, 1979, 280, 60-62.

RitTen, S. M. The vertical-horizontal illusion. Psychological Monographs, 1917, 23, No. 101

Schiller, P. H., Finlay, B. L., \& Volman, S. F. Quantitative analysis of single-cell properties in monkey striate cortex. II. Orientation specificity and ocular dominance. Journal of Neurophysiology, 1976, 39, 1320-1333.

Seruler, R. W., Rubin, E. L., \& Cushman, W. H. Selectivities of human visual mechanisms for direction of movement and contour orientation. Journal of the Optical Saciety of America, $1968,58,1146-1150$.

Sharpe, C. R., \& Tolhunst, D. J. Orientation and spatial frequency channels in peripheral vision. Vision Research, 1973, 13, 2103-2112.

TAYLon, M. M. Visual discrimination and orientation. Journal of the Optical Society of America, 1963, 53, 763-765.

Thompson, J., \& Schifrman, H. R. The effect on the magnitude of the horizontal-vertical illusion of horizontal retinal eccentricity. Vision Research, 1974, 14, 1463-1465.
Virsu, V. Dark adaptation shifts apparent spatial frequency. Vision Research, 1974, 14, 433-435.

Virsu, V., \& Nyman, G. Monophasic temporal modulation increases apparent spatial frequency. Perception, 1974, 3, 337-353.

WENDERoTh, $\mathrm{P}$. A possible confounding between the orthogonal perceived spatial frequency shift and the vertical-horizontal illusion. Vision Research, 1980, 20, 1021.

\section{NOTES}

1. A complete description of the calibration procedures used may be obtained by writing the author. A description may also be found in Bowker (1980a).

2. Observer D.O.B. (the author) was refracted and found to be a well-corrected myope ( -3.0 diopters O.D., -1.75 diopters O.S.) with a slight corrected astigmatism ( -0.25 diopters at $110 \mathrm{deg}$ ) in the right eye.

3. The laser interferometer was initially constructed by Martin Greenfield as part of his dissertation requirements. Several minor changes, including the second channel, were introduced into the system by the author. I gratefully acknowledge Dr. Greenfield's assistance on this aspect of the experiment.

4. Observer A.N.P. was a naive undergraduate volunteer. This observer was also run in the experiment reported in the the preceding paper (Bowker, 1981) and was found to have a significant orientation bias in his grating and line choices. Along with the majority of subjects reported in that experiment, A.N.P. chose horizontal gratings as coarser than vertical gratings most of the time. A.N.P. reported having normal uncorrected vision (recently tested).

\section{APPENDIX}

\section{Analysis of Spatial Frequency Matching Data}

The spatial frequency matching data presented in Experiment 1 were collapsed to form several scales of apparent spatial frequency as a function of orientation. This normalization to the vertical is based upon a linear model that assumes that spatial frequency match deviations between different orientations result from meridional differences in apparent spatial frequency. This model may be explicitly stated in terms of three primary variables. The first primary (P1) represents the difference in spatial frequency appearance between a vertical grating and a 45-deg grating; the second primary (P2) is the apparent frequency difference between a vertical grating and a horizontal grating; and the third primary (P3) is the difference between a vertical grating and a 135-deg grating. All frequency matches are modeled on the basis of these three primaries. Normalization of the data to the vertical was essentially an attempt to describe these primaries. The following equations were used in the normalization process:

$$
\begin{aligned}
& \mathrm{SF}_{\mathrm{dev}}\left(0^{\circ}, 45^{\circ}\right)=\mathrm{SF}_{\mathrm{dev}}\left(0^{\circ}, 90^{\circ}\right)-\mathrm{SF}_{\mathrm{dev}}\left(45^{\circ}, 90^{\circ}\right) \\
& =\operatorname{SF}_{\mathrm{dev}}\left(0^{\circ}, 135^{\circ}\right)-\mathrm{SF}_{\mathrm{dev}}\left(45^{\circ}, 135^{\circ}\right) \\
& \operatorname{SF}_{\mathrm{dev}}\left(0^{\circ}, 90^{\circ}\right)=\operatorname{SF}_{\mathrm{dev}}\left(0^{\circ}, 45^{\circ}\right)+\mathrm{SF}_{\mathrm{dev}}\left(45^{\circ}, 90^{\circ}\right) \\
& =\mathrm{SF}_{\mathrm{dev}}\left(0^{\circ}, 135^{\circ}\right)-\mathrm{SF}_{\mathrm{dev}}\left(90^{\circ}, 135^{\circ}\right) \\
& \mathrm{SF}_{\mathrm{dev}}\left(0^{\circ}, 135^{\circ}\right)=\mathrm{SF}_{\mathrm{dev}}\left(0^{\circ}, 45^{\circ}\right)+\mathrm{SF}_{\mathrm{dev}}\left(45^{\circ}, 135^{\circ}\right) \\
& =\mathrm{SF}_{\mathrm{dev}}\left(0^{\circ}, 90^{\circ}\right)+\mathrm{SF}_{\mathrm{dev}}\left(90^{\circ}, 135^{\circ}\right)
\end{aligned}
$$

where $\mathrm{SF}_{\mathrm{dev}}$ is the spatial frequency match deviation be- 
Table Al

\begin{tabular}{rrrrr}
\multicolumn{2}{c}{$\begin{array}{c}\text { Test/Standard } \\
\text { Orientations }\end{array}$} & P1 & P2 & P3 \\
\hline 0 & 0 & 0 & 0 & 0 \\
0 & 45 & 1 & 0 & 0 \\
0 & 90 & 0 & 1 & 0 \\
0 & 135 & 0 & 0 & 1 \\
45 & 45 & 0 & 0 & 0 \\
45 & 90 & -1 & 1 & 0 \\
45 & 135 & -1 & 0 & 1 \\
90 & 90 & 0 & 0 & 0 \\
90 & 135 & 0 & -1 & 1 \\
135 & 135 & 0 & 0 & 0 \\
\hline
\end{tabular}

tween gratings presented in the orientations given in parentheses. These equations can be thought of as one dimensional vector representations of the spatial frequency match deviations. Using these equations, it is possible to express any orientation combination in terms of the three primary variables. For instance, $\mathrm{SF}_{\mathrm{dev}}\left(45^{\circ}, 90^{\circ}\right)$ may be expressed in terms of the first and second primaries. Since

$$
\mathrm{SF}_{\mathrm{dev}}\left(0^{\circ}, 45^{\circ}\right)=\mathrm{SF}_{\mathrm{dev}}\left(0^{\circ}, 90^{\circ}\right)-\mathrm{SF}_{\mathrm{dev}}\left(45^{\circ}, 90^{\circ}\right)
$$

then

$$
\mathrm{SF}_{\mathrm{dev}}\left(45^{\circ}, 90^{\circ}\right)=\mathrm{SF}_{\mathrm{dev}}\left(0^{\circ}, 90^{\circ}\right)-\mathrm{SF}_{\mathrm{dev}}\left(0^{\circ}, 45^{\circ}\right)
$$

Therefore, $\mathrm{SF}_{\mathrm{dev}}\left(45^{\circ}, 90^{\circ}\right)$ equals $\mathrm{P} 2-\mathrm{P} 1$. A similar procedure can be used to express all of the match deviations in terms of the three primary variables.

To test this linear model, a regression analysis was performed using the general linear model of the SAS statistical package. The three primary variables were used as regression variables in the model and were assigned values shown in Table Al according to orientation matching condition. Other variables were included in the regression analysis to account for the spatial frequency of the standard, the interaction of this factor with the three orientation primaries, and the effect of standard side on the match deviations. The linear model took the following form:

$$
\begin{aligned}
S_{\text {dev }}= & a_{0}+a_{1} P 1+a_{2} P 2+a_{3} P 3+a_{4} S F+a_{5}(P 1 * S F) \\
& +a_{6}(P 2 * S F)+a_{7}(P 3 * S F)+a_{8}(\text { side }),
\end{aligned}
$$

where SF represents the spatial frequency of the standard and side represents the side of the standard grating.

A regression analysis of the spatial frequency matching data of Observer D.O.B. using this model accounted for $59.28 \%$ of the variance. All sources of variance were highly significant except for the main factor of spatial frequency. This factor did interact significantly with all orientation factors. Data entered into the linear model consisted of single trial observations and were not entered as averages of several trials. Results of the regression analysis are provided in Table A2.

Individual analyses were carried out for each of the four standard spatial frequencies to derive the coefficients of the three primary variables. These coefficients are provided for each frequency in Table A3. These values are very similar to the averages plotted in Figure 3. As with the values plotted in Figure 3, these coefficients represent percent spatial frequency match deviations. All coefficients are significant with a $\mathrm{p}<.0001$ except for ${ }^{*}$, which had a $\mathrm{p}<.0014$.

Table A3

\begin{tabular}{cccc}
\hline & \multicolumn{3}{c}{ Primary } \\
\cline { 2 - 4 } Spatial & P1 & P2 & P3 \\
\hline 95 & -1.39 & -2.16 & $-.62 \dagger$ \\
4.00 & -.79 & -2.34 & -.61 \\
8.00 & +1.92 & -2.38 & +.94 \\
12.00 & +4.65 & -4.05 & +2.08 \\
\hline
\end{tabular}

\begin{tabular}{|c|c|c|c|c|c|c|}
\hline Source & df & SS & MSe & F & Percent of Variance & $\mathrm{p}<$ \\
\hline Model & 8 & 15926.65 & 1990.83 & 522.34 & 59.28 & .0001 \\
\hline P1 & 1 & 3246.84 & & 851.88 & 12.09 & .0001 \\
\hline $\mathbf{P 2}$ & 1 & 7151.53 & & 1876.36 & 26.62 & .0001 \\
\hline P3 & 1 & 103.74 & & 27.22 & .39 & .0001 \\
\hline SF & 1 & 3.96 & & 1.04 & .01 & .3084 \\
\hline $\mathrm{P} 1 * \mathrm{SF}$ & 1 & 3731.31 & & 978.99 & 13.89 & .0001 \\
\hline $\mathrm{P} 2 * \mathrm{SF}$ & 1 & 1040.03 & & 272.87 & 3.87 & .0001 \\
\hline P3*SF & 1 & 314.02 & & 82.39 & 1.17 & .0001 \\
\hline Side & 1 & 335.24 & & 87.96 & 1.25 & .0001 \\
\hline Error & 2870 & 10938.69 & 3.81 & & & \\
\hline Total & 2878 & 26865.34 & & & & \\
\hline
\end{tabular}

Note-All coefficients are significant at $p<.0001$ unless otherwise indicated. ${ }^{*} \mathrm{cpd}$. $t_{p}<.0014$.

Table A2

(Received for publication December 29, 1980; revision accepted April 14, 1981.) 